\title{
Alternative split sites for fragment complementation, and glyphosate function as extra ligand and stabilizer for the AroA enzyme complexes
}

\author{
LI Yan ${ }^{1}$, SUN YiCheng ${ }^{1,2}$, YAN HaiQin $^{1} \&$ WANG YiPing ${ }^{1 *}$ \\ ${ }^{1}$ National Key Laboratory of Protein Engineering and Plant Genetic Engineering, College of Life Sciences, Peking University, Beijing 100871, China; \\ ${ }^{2}$ Institute of Pathogen Biology, Chinese Academy of Medical Sciences and Peking Union Medical College, Beijing 100730, China
}

Received September 19, 2010; accepted December 10, 2010

\begin{abstract}
Protein reconstitution analysis can be useful for studies on protein evolution, protein folding and macromolecular assembly. AroA is a key enzyme in the pathway toward the synthesis of aromatic amino acids in microorganisms and plants, and is the target of the herbicide glyphosate. Our previous study showed that functional AroA enzyme from Escherichia coli could be reconstituted from two 220 -amino acid fragments of the protein. In this study, we explored this fragment complementation of AroA. Through a systematic study of fragment complementation, we show that successful fragment complementation can be achieved in vivo, when the split sites are within secondary structure elements as well as at loops between structure elements. In addition, we provide evidence, for the first time, that extra ligand, such as glyphosate, can function as a stabilizer of the reconstituted complexes in vivo. Therefore, our results may provide important implications for protein evolution and complex assemblies.
\end{abstract}

AroA, fragment complementation, glyphosate, extra ligand, complex stability

Citation: $\quad$ Li Y, Sun Y C, Yan H Q, et al. Alternative split sites for fragment complementation, and glyphosate function as extra ligand and stabilizer for the AroA enzyme complexes. Chinese Sci Bull, 2011, 56: 514-520, doi: 10.1007/s11434-010-4323-9

Proteins reconstituted from fragments produced by proteolytic, chemical, or genetic methods have been studied for the last 50 years [1-6]. A successful reconstitution implies that interchain packing interactions within the protein are specific enough to allow the fragments to form the nativelike structure. Protein reconstitution has been used to study protein evolution, protein folding, macromolecular assembly, and mapping contacts in membrane-embedded proteins $[7,8]$. However, little systematic exploration of the scope of protein fragment complementation has been done.

5-enolpyruvylshikimate-3-phosphate (EPSP) synthase (AroA) is a key enzyme involved in aromatic amino acid biosynthesis in bacteria, fungi, and higher plants, and is the target of the global herbicide glyphosate $[9,10]$. AroA catalyzes the reversible condensation of shikimate-3-phosphate (S3P) and phosphoenolpyruvate (PEP) to yield EPSP and inorganic phosphate [10]. Glyphosate, an analog of PEP,

*Corresponding author (email: wangyp@pku.edu.cn) binds to the binding site for PEP on the AroA enzyme and blocks enzyme activity by forming a more stable ternary complex with AroA and the other substrate, S3P (AroA·S3P·glyphosate) [11]. Glyphosate tolerant AroA can be obtained by a G96A substitution in the aroA gene of Escherichia coli, and in this case, glyphosate can no longer bind this mutant to inhibit its enzyme activity $[12,13]$.

AroA consists of two distinct globular domains and each domain contains 3 copies of a $\beta \alpha \beta \alpha \beta \beta$-folding unit [14]. The domains maintain an open conformation in the absence of substrates, whereas binding of S3P in combination with PEP or glyphosate to AroA induces a conformational change into a closed stage $[11,15]$.

Our previous study showed that functional AroA could be reconstituted from two fragments of the protein corresponding to a.a. 1-218 and 219-427 [16]. These fragments result from a split site located in the loop between two $\beta$ strands (Table 1). To test whether this split site is unique for fragment complementation of AroA, we systematically 
Table 1 Reconstitution of AroA activity by fragment complementation

\begin{tabular}{|c|c|c|c|c|c|c|}
\hline \multicolumn{2}{|c|}{ EPSPS fragments $^{\text {a) }}$} & \multirow{2}{*}{ Split region $^{\text {b) }}$} & \multicolumn{3}{|c|}{ Complementation $^{\mathrm{c})}$} & \multirow{2}{*}{ Growth rates $^{\mathrm{d}}$} \\
\hline $\mathrm{N}$-term & C-term & & N-term & C-term & $\mathrm{N}+\mathrm{C}$ & \\
\hline N85 & $\mathrm{C} 86$ & Loop between folding units & - & - & + & $98+9$ \\
\hline N104 & $\mathrm{C} 105$ & $\alpha$ helix & - & - & - & $l^{\mathrm{e})}$ \\
\hline $\mathrm{N} 154$ & $\mathrm{C} 155$ & Loop between folding units & - & - & + & $91+7$ \\
\hline N165 & $\mathrm{C} 166$ & Loop between $\alpha$ helix $\& \beta$ strand & - & - & + & $399+35$ \\
\hline N182 & $\mathrm{C} 183$ & $\beta$ strand & - & - & + & $605+47$ \\
\hline $\mathrm{N} 218$ & $\mathrm{C} 219^{\mathrm{f}}$ & $\alpha$ helix & - & - & + & $101+8$ \\
\hline $\mathrm{N} 224$ & $\mathrm{C} 225$ & $\beta$ strand & - & - & + & $105+8$ \\
\hline $\mathrm{N} 227$ & $\mathrm{C} 228$ & Loop between folding unit & - & - & + & $88+8$ \\
\hline $\mathrm{N} 234$ & $\mathrm{C} 235^{\mathrm{f}}$ & Loop between folding unit & - & - & - & / \\
\hline $\mathrm{N} 238$ & $\mathrm{C} 239$ & $\beta$ strand & - & - & - & / \\
\hline $\mathrm{N} 240$ & $\mathrm{C} 241$ & Loop between domains & - & - & - & / \\
\hline $\mathrm{N} 245$ & $\mathrm{C} 246$ & $\alpha$ helix & - & - & - & I \\
\hline $\mathrm{N} 298$ & C299 & Loop between folding unit & - & - & + & $95+9$ \\
\hline N371 & C372 & Loop between folding unit & - & - & + & $85+10$ \\
\hline N384 & C385 & Loop between $\alpha$ helix $\& \beta$ strand & - & - & - & l \\
\hline \multicolumn{2}{|c|}{ Intact AroA } & & & & & $85+6$ \\
\hline
\end{tabular}

a) DNA fragments producing amino-terminal and carboxyl-terminal fragments were cloned into pACYC184 and pBR322, respectively. b) The splits sites were chosen based on the structure of AroA. c) Cells harboring different AroA fragments were tested for growth on the M63 agar plates. d) Cells harboring different AroA fragments were tested for growth in M63 liquid medium. e) "/" means not determined. f) N218/C228 and N234/C235 have been interpreted previously. +, Reconstitution of AroA activity happened by fragment complementation; -, reconstitution could not be determined.

tested the complementation of protein fragments created by split sites in other parts of the protein. In our study we found that AroA activity reconstitution could be achieved, when the split sites are located at loops between folding units, or within secondary structure elements, or at loops between secondary structure elements. Moreover, it was observed that stability of the reconstituted enzyme complexes, but not of the intact counterpart, can be enhanced by the presence of moderate levels of herbicide glyphosate, as extra ligand in vivo. Therefore, our results may provide important implications for the evolution and assembly of proteins and complexes.

\section{Materials and methods}

\subsection{Medium and chemicals}

S3P (barium) was a gift from Prof. Nikolaus Amrhein (ETH, Zurich, Switzerland). Glyphosate free acid form and phosphoenolpyruvate (PEP) were purchased from Sigma. For testing complementation in vivo cells were grown on M63 minimal medium supplemented with $0.4 \%$ glucose as carbon source, plus appropriate antibiotics [17].

\subsection{Strain and plasmid constructions}

An aroA deletion strain, BD2100, was constructed from $E$. coli B121(DE3) by allelic replacement using pKO3 plasmid as described previously [18].
All strains and plasmids used in this study are listed in supplementary data.

\subsection{Growth rates}

E. coli BD2100 harboring plasmids were inoculated into 25 mL M63 minimal medium with an initial $A_{600}$ of approximately 0.02 . Cells were grown with shaking at $37^{\circ} \mathrm{C}$ and their densities were measured at $A_{600}$.

\subsection{Preparation of crude enzymes}

Crude enzymes were prepared as described previously $[16,17]$. Cells containing different plasmids were grown in $300 \mathrm{~mL}$ LB broth or M63 minimal medium supplemented with glyphosate at various concentrations to a cell density of $A_{600} 2.0$. The cell pellets were collected and re-suspended in $30 \mathrm{~mL}$ buffer A (50 mmol/L Tris buffer ( $\mathrm{pH} \mathrm{7.8),}$ $0.4 \mathrm{mmol} / \mathrm{L}$ dithiothreitol). After lysis and centrifugation, ammonium sulfate was added to the supernatants. The fractions between $50 \%$ and $70 \%$ saturation of ammonium sulfate were collected and dialysed overnight in buffer A.

\subsection{Western blot analysis of AroA fragments}

Western blot analysis was performed as described previously [16]. Briefly, proteins were applied to a $16 \%$ SDS/polyacrylamide gel, and separated polypeptides were analyzed using immunoblots. The immunoblots were 
probed with 1:2000 dilution of AroA rabbit polyclonal antibody [16]. The antibody-antigen complex was visualized with alkaline phosphatase conjugated to goat anti-rabbit IgG (Promega).

\subsection{Enzyme assay}

AroA enzyme activity was determined by measuring phosphate release using the malachite green dye assay method as described previously [17]. Briefly, the standard reaction was carried out at $28^{\circ} \mathrm{C}$ in a final volume of $50 \mu \mathrm{L}$ containing 50 mmol/L HEPES (pH 7.0), $1 \mathrm{mmol} / \mathrm{L} \mathrm{S3P}, 1 \mathrm{mmol} / \mathrm{L}$ PEP, and the crude enzyme. After incubation for 1-10 min, 800 $\mathrm{mL}$ of malachite green-ammonium molybdate colorimetric solution was added, and $1 \mathrm{~min}$ later, $0.1 \mathrm{~mL}$ of a $34 \%$ sodium citrate solution was added to stop the reaction. After 30-min incubation at room temperature, absorbances of samples were measured at $660 \mathrm{~nm}$. In this case, the same reaction solution without $\mathrm{S} 3 \mathrm{P}$ was used as the zero control.

\section{Results}

\subsection{Structural based functional assembly of split AroA}

Protein reconstitution resulting from the conversion of nonfunctional monomers to functional heterodimers is a reverse of the evolutionary process in which functional structural elements or domains are recruited and then fused at the genetic level. It has been suggested that AroA had evolved from 6-fold replication of a primordial gene for the folding unit [15], which suggests that reconstitution of AroA might be achieved when the split site is located within junctions of folding units. To test this hypothesis, 6 split sites at the loop between folding units were chosen based on the known crystal structure of E. coli AroA [16]. We also chose another 11 sites based on their structural locations (including the 2 sites tested previously): 1 at the loop between domains, 5 at the loop between $\alpha$ helix and $\beta$ strands, and another 5 within $\alpha$ helix or $\beta$ strands (Figure 1 and Table 1).

The resulting amino-terminal and carboxyl-terminal portion of each pair was cloned downstream of the constitutive $\operatorname{Tet}^{\mathrm{r}}$ promoter and expressed from plasmids pACYC184 and pBR322 respectively. In vivo activities of these fragments were individually tested by introducing each plasmid into $E$. coli aroA deletion strain BD2100. None of the plasmids encoding either the amino- or carboxyl-terminal fragments alone could support growth of E. coli aroA deletion strain BD2100 on the M63 minimal medium, indicating that neither fragment alone has AroA activity.

We next tested for assembly of AroA enzyme activity, by introducing pairs of amino- and carboxyl-terminal plasmids together into BD2100. We observed that 11 out of the introduced 17 pairs were active, and could support growth of

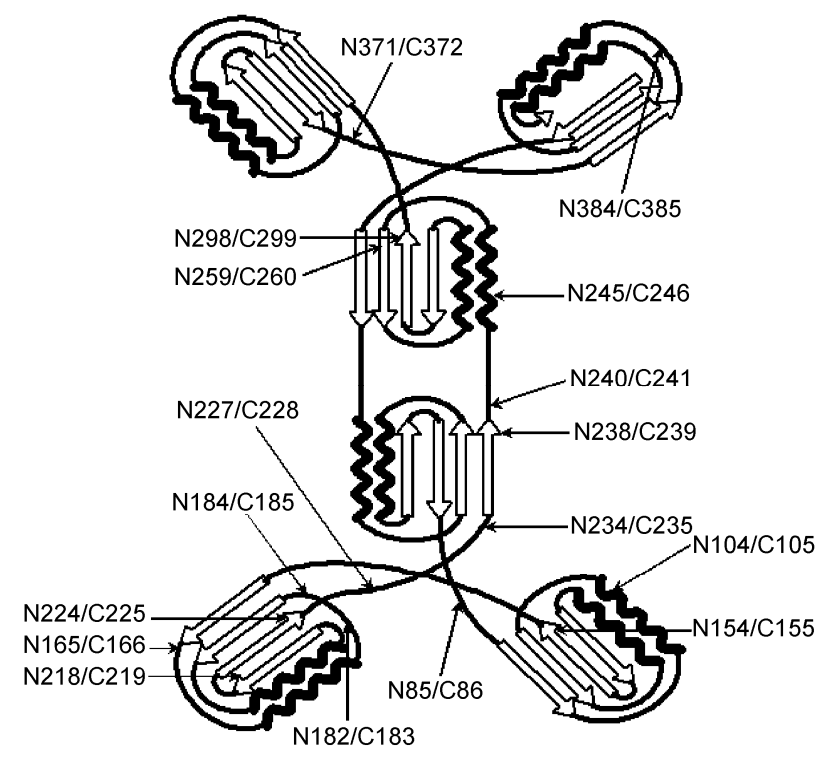

Figure 1 The location of spit sites. Locations of split sites are indicated in the schematic illustration of the chain tracing of E. coli AroA [15].

the complementing strain on M63 minimal medium.

In order to eliminate the possibility that endogenous intact enzyme may have been present in the cells, which may lead to the AroA activity, proteins from cells among these 11 active split pairs of recombinants were analyzed with SDS/polyacrylamide gel electrophoresis and subsequent western blotting analysis against AroA antibodies. The results show that AroA fragments with expected sizes could be observed in most cases and more that no intact AroA enzyme could be detected in any of the cells (Figure 2). Therefore any AroA activity in these transformed cells was due to reconstitution from the introduced fragments and not due to any intact AroA activity.

Under native conditions, gel filtration of the complementing extracts of cells containing the active split pairs showed that AroA enzyme activity migrated at the identical position as that of the intact, monomeric wild-type enzyme (data not shown). This result is consistent with our previous report on the N218/C219 pair [16], which indicates they all can physically form active complexes in a "hetrodimeric" manner, which mimics the intact wild-type enzyme.

\subsection{Ligand mediated enhancement in the stability of complementation complexes}

These split sites of AroA were further characterized by measuring growth rates of E. coli aroA mutant BD2100 harboring pairs of the appropriate plasmids in M63 minimal medium. The results showed that BD2100 strains that contain N85/C86, N154/C155, N227/C228, N298/C299 or N371/C372 pairs, which were split at the loop of folding units, grow much better than strains harboring the other 6 
pairs. In this case, during exponential growth phases, their doubling time is less than 100 min (ranging from 85 to 98 $\mathrm{min})$, which is similar to that of their intact counterpart (85 min), and little better than that of previously identified N218/C219 pair (101 min, Table 1).

To further test the above result, enzyme activities of N85/C86, N154/C155, N227/C228, N298/C299 and N371/ C372 complexes were assayed, in parallel with western blotting assays. In this case, enzyme complexes were partially purified from cells grown in LB medium. To our surprise, N85/C86 and N227/C228 showed some, but less enzyme activity than that of N218/C219, whereas N154/C155, N298/C299 and N371/C372 showed no enzyme activity (Table 2). Consistent with no detectable enzyme activity in the assays, fragments containing C155, C299 or C372 could not be detected by Western blotting (Figure 3 ).

To investigate if the effect observed above could be af- fected by growth medium, similar assays were carried out when cells were grown in M63 minimal medium. The result showed that except N154/C155 and N371/C372 pairs, which showed very little enzyme activity in LB rich medium, a general enhancement in enzyme activity was observed for the other four pairs of enzyme complexes as well as their intact counterpart. However, to our surprise, the enhancements observed are not to the same extent among different samples. We observed that the enhancement became more drastic for some complementing complexes than that of the intact counterpart. For instance, the activity of N298/299 complex increased almost 10-fold when cells grown in M63 minimum medium than that in LB rich medium. In contrast, for the intact enzyme, only a 1.9-fold enhancement was observed (Table 2).

Many reasons could account for the results observed above. However, the fact that when $E$. coli cells grown in

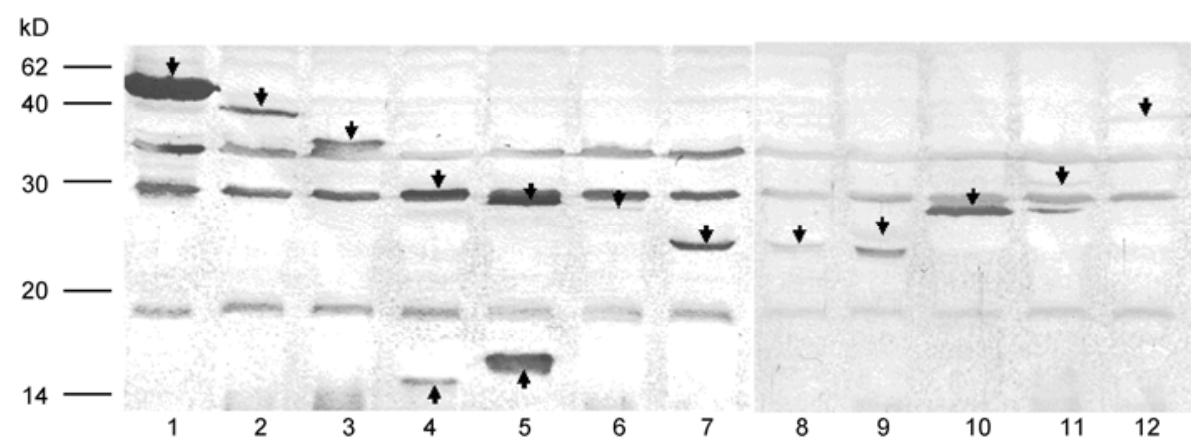

Figure 2 Western blot analysis of AroA fragments that were produced in complemented cells. Intact AroA (lane 1), N85/C86 (lane 2), N104/C105 (lane 3), N154/C155 (lane 4), N165/C166 (lane5), N184/C185 (lane 6), N218/C219 (lane 7), N224/C225 (lane 8), N227/C228 (lane 9), N259/C260 (lane 10), N298/C299 (lane 11) and N371/C372 (lane 12) grown in LB medium. Locations of size markers are indicated at the left. Fragments of the expected size are indicated by filled arrowhead.

(a)

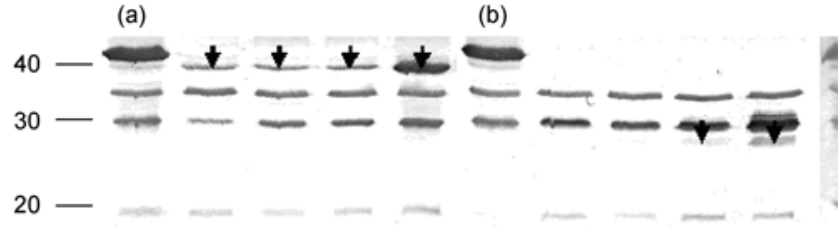

14 (c)

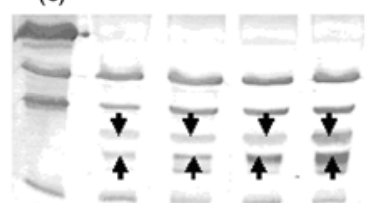

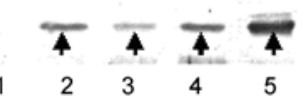

(e)

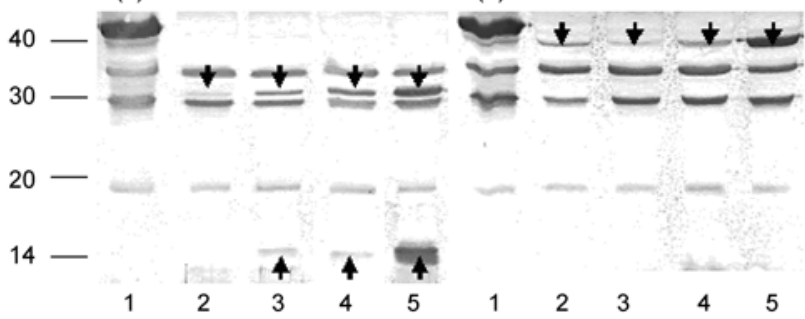

Figure 3 Western blot analysis of AroA fragments that were produced at different conditions. N85/C86 (a), N154/C155 (b), N227/C228 (c), N298/C299 (d) and N371/C372 (e) grown in LB medium (lane 2), M63 medium (lane 3), M63 medium with 1 mmol/L glyphosate (lane 4) and M63 medium with 10 $\mathrm{mmol} / \mathrm{L}$ glyphosate (lane 5). Intact AroA grown in LB medium (lane 1) used as control. Locations of size markers are indicated at the left. Fragments of the expected size are indicated by filled arrowhead. 
Table 2 Specific enzyme activity of intact- or reconstituted- AroA under different gorwth conditions ${ }^{\text {a) }}$

\begin{tabular}{ccccc}
\hline \multirow{2}{*}{ Enzyme } & \multicolumn{3}{c}{ Specific activity (nkat/mg protein) $)^{\mathrm{b})}$} & M63 (10 mmol/L GLP) \\
\cline { 2 - 5 } & $\mathrm{LB}$ & \multicolumn{1}{c}{$\mathrm{M} 63$} & $\mathrm{M} 63(1 \mathrm{mmol} / \mathrm{L}$ GLP $)$ & $22.40 \pm 4.96$ \\
Intact & $8.40 \pm 1.51$ & $24.21 \pm 3.46$ & $23.36 \pm 2.98$ & $4.46 \pm 1.20$ \\
N85/C86 & $0.41 \pm 0.14$ & $1.28 \pm 0.21$ & $1.71 \pm 0.15$ & $1.64 \pm 0.38$ \\
N154/C155 & $0.01 \pm 0.01$ & $0.06 \pm 0.01$ & $0.16 \pm 0.03$ & c) $^{\mathrm{c}}$ \\
N218/C219 & $0.46 \pm 0.20$ & $1.63 \pm 0.39$ & $2.22 \pm 0.34$ & $8.18 \pm 2.31$ \\
N227/C228 & $0.36 \pm 0.16$ & $1.33 \pm 0.38$ & $1.87 \pm 0.30$ & $5.65 \pm 1.34$ \\
N298/C299 & $0.02 \pm 0.01$ & $0.03 \pm 0.01$ & $0.44 \pm 0.10$ & $2.75 \pm 0.35$ \\
Intact G96A & $2.31 \pm 0.28$ & $3.29+0.21$ & $2.83 \pm 0.12$ & $0.69 \pm 0.12$ \\
N218/C219-G96A & $0.15 \pm 0.03$ & $1.03+0.13$ & $0.82 \pm 0.10$ & $0.72 \pm 0.09$ \\
N227/C228-G96A & $0.14 \pm 0.02$ & $1.17 \pm 0.11$ & $0.83 \pm 0.15$ & \\
\hline
\end{tabular}

a) The results presented are an average of two sets of experiments done in triplicate. AroA fragments produced from complemented cells grew in LB medium, M63 medium, M63 medium with $1 \mathrm{mmol} / \mathrm{L}$ glyphosate and M63 medium with $10 \mathrm{mmol} / \mathrm{L}$ glyphosate. b) Specific activity was determined at $1.0 \mathrm{mmol} / \mathrm{L}$ PEP and $1.0 \mathrm{mmol} / \mathrm{L}$ S3P. c) Cells harboring N218/C219 can not grow in M63 medium with $10 \mathrm{mmol} / \mathrm{L}$ glyphosate.

LB rich medium do not need to synthesize as much aromatic amino acids as that of in M63 minimum medium, and the fact that S3P and PEP are substrates of the AroA enzyme and therefore can be used as extra ligand for the complementing enzyme complexes, one could propose that higher concentrations of ligands (such as S3P and PEP) may play a role in the drastic enhancement of enzyme activities observed for the complementing pairs.

In order to verify the above hypothesis, we took the advantage that the herbicide glyphosate, as an analogue of PEP, can also bind to the AroA enzyme in the presence of S3P [11], and thus can be used as an extra ligand in an artificial system. In this case, similar assays were carried out when cells were grown in M63 minimum medium in presence of moderate concentrations of glyphosate. The results showed that when moderate levels of glyphosate were provided in the growth medium, glyphosate-dependent drastic enhancements of enzyme activities were observed (Table 2). The enhancement in enzyme activities can be described as below: First, the enhancement is specific for complementing pairs, but not for the intact counterpart. Second, the enhancement was bigger in $10 \mathrm{mmol} / \mathrm{L}$ than in $1 \mathrm{mmol} / \mathrm{L}$ glyphosate, indicating that the glyphosate-dependent enhancement of enzyme activities is dose-dependent (Table 2). Third, a trend in enzyme activities of the complementing pairs in the function of growth media could be observed as M63+10 $\mathrm{mmol} / \mathrm{L}$ glyphosate $>\mathrm{M} 63+1 \mathrm{mmol} / \mathrm{L}$ glyphosate> M63>LB. In parallel, Western blotting analysis also showed that all fragments except $\mathrm{C} 373$ (which is too small to be detected) increased their expression in the pre- sence of glyphosate. A similar trend in protein fragment concentrations as that in enzyme activities in the function of growth mediums could be observed, i.e. M63 + $10 \mathrm{mmol} / \mathrm{L}$ glyphosate $>$ M63+1 mmol/L glyphosate $>$ M63 $>$ LB (Figure 3). In contrast, enzyme activities of the intact counterpart did not follow the same trend (Table 2).

If glyphosate could be used as an extra ligand to stabilize the complementing enzyme complexes in vivo, then one would expect that glyphosate can not function as stabilizer when it is not bound to AroA. In this case, we took advantage of a mutant of AroA, AroA-G96A, which cannot bind glyphosate (glyphosate tolerant), but to some extent, retained its enzyme activity [12,13]. It was shown that split fragments of this mutant AroA, N218/C219-G96A and N227/C228-G96A, can reconstitute the enzyme activity in a glyphosate tolerant manner [16] (Li et al., unpublished results). We tested these fragment pairs along with intact AroA-G96A, for enzyme activity in our assays. As expected, none of the pairs showed enhanced enzyme activities in the presence of glyphosate in the growth medium (Table 2). Therefore, there is a direct correlation between glyphosate binding and enhancement of enzyme activities of the complementing complexes.

Taken together, the above results demonstrate that extra ligand (such as glyphosate) can function as a stabilizer for the complementing enzyme complexes in vivo.

\section{Discussion}

In this study, we systematically explored the scope of fragment complementation in the reconstitution of activity of the AroA enzyme. The successful reconstitution of active enzyme activity from 11 fragment pairs suggests that the internal complementarity is generally sufficient to overcome breaks in the peptide backbone to allow assembly of a functional structure. The locations of cleavage sites that allow successful reconstitution can be grouped according to the position of the split sit in relation to the structural features of the crystal structure determined AroA protein; Group I fragments were split within $\beta$ strands, group II fragments were split at the loop between secondary structures and group III fragments were split within the loops of folding units. The most successful fragment complementation occurred with fragments with group III split sites. In these cases, as each of the fragments is a complete folding unit by itself, it is expected that they can be properly folded respectively, which may result in better complementation. This is 
supported by the fact that BD2100 containing these AroA fragment pairs grow very well in M63 media, that is, in all cases with a doubling time of less than $100 \mathrm{~min}$ (Table 1).

The reconstituted AroA complexes showed higher enzyme activities when cells were grown in M63 minimum medium than in LB rich medium (Table 2). It is known that in the shikimate pathway, the enzyme responsible for synthesizing S3P is de-repressed 10-fold when cells are starved for tryrosine and tryptophan or the try $R$ regulatory gene is inactivated in the genome [19]. Therefore, one would expect that higher concentrations of S3P exist in M63 minimum medium than in LB rich medium. The extra S3P in M63 grown cells could be used as extra ligand, and hence as stabilizer for a reconstituted AroA complexes. Such a scenario would account for the observed activity measured for cells containing the various fragment pairs (Table 2). Glyphosate, an analogue of PEP, can also bind to AroA and is expected to act as extra ligand to stabilize the reconstituted complex. This notion is supported by the fact that addition of moderate levels of glyphosate to the growth media also resulted in higher activities for the reconstituted AroA complexes, but not for the intact AroA enzyme in vivo (Table 2). When enzyme was prepared by dialysis of crude enzyme, glyphosate did not bind to enzyme. Since glyphosate is an inhibitor of AroA, if it were still bound to reconstituted complexes, one would expect that enzyme activity would decrease. The fact is that higher activities were observed for the reconstituted AroA complexes when moderate levels of glyphosate were added to the growth media. One could propose that glyphosate may be enhancing the stability of reconstituted enzyme complexes in vivo, thus increasing the quantity of reconstituted enzymes in the crude extracts, which in turn, results in enhanced enzyme activities observed for the reconstituted complexes. This hypothesis is further supported by the Western blot results that show that more target protein fragments were produced with the moderate levels of glyphosate present in the medium (Figure 3). In contrast, when a mutated form of AroA enzyme, which can no longer bind glyphosate was used in the assay, addition of glyphosate to the growth media no longer enhanced the activity of the complex (Table 2). Therefore, our results demonstrate that glyphosate can stabilize the reconstituted complex, only when it was used as ligand.

AroA dissociated fragments may be stabilized by extra ligand. The reconstituted AroA complex is in a dynamic equilibrium of associated and dissociated fragments. Dissociated fragments may be considered an abnormal structure for the complex and hence, are likely to be subjected to degradation by proteases [20], which may account for the instability of reconstituted AroA in vivo. Extra ligand, such as glyphosate, may bring the complementing fragments closer together, to a conformation similar to that of their intact counterpart. In this case, extra ligand could mediated conformational change from "open stage" to "closed stage", as that observed in crystal structures for their intact coun- terpart $[11,15]$, which in turn, may increase the strength of the interactions between the complementing fragments, and shift the equilibrium towards associated complex, and thus prevent the AroA fragments from degradation. This is the first time someone has shown extra ligand can stabilize reconstituted complex in vivo.

Successful reconstitution in vivo requires that internal complementarity be highly specific to overcome competing interactions with other protein sequences. Non-covalent interactions such as hydrogen bonding, electrostatic interactions and especially hydrophobic forces contribute significantly to the specificity of internal complementarity [21-25]. These interactions govern a protein's native structure, and have been optimized through evolution, yielding a strong preference for the native packing of structural elements. The dissociated sub-domains may overcome competing interactions with other protein sequences, and find a way to spontaneously assembly to the native structure. Our results may have implications for the evolution and assembly of large proteins and complexes starting from specific non-covalent interactions between individual structure elements.

We thank Prof. Nikolaus Amrhein (ETH, Zurich, Switzerland) for sending us S3P substrate. We thank Dr. Karen Yook for critical reading of the manuscript. This work was supported by the National Transgenic Research and Industrialization Special Foundation of China (2009ZX08009057B and 2008ZX08009-003-002), and the National Hi-Tech Research and Development Program of China (2007AA021307).

1 Richards F M. On the enzymic activity of subtilisin-modified ribonuclease. Proc Natl Acad Sci USA, 1958, 44: 162-166

2 Taniuchi H, Anfinsen C B, Sodja A. Nuclease-T: An active derivative of staphylococcal nuclease composed of two noncovalently bonded peptide fragments. Proc Natl Acad Sci USA, 1967, 58: 1235-1242

3 Bibi E, Kaback H R. In vivo expression of the lacY gene in two segments leads to functional lac permease. Proc Natl Acad Sci USA, 1990, 87: 4325-4329

4 Shiba K, Schimmel P. Functional assembly of a randomly cleaved protein. Proc Natl Acad Sci USA, 1992, 89: 1880-1884

5 Berggard T, Thulin E, Akerfeldt K S, et al. Fragment complementation of calbindin D28k. Protein Sci, 2000, 9: 2094-2108

6 Braun M, Endriss F, Killmann H, et al. In vivo reconstitution of the FhuA transport protein of Escherichia coli K-12. J Bacteriol, 2003, 185: 5508-5518

7 Hakansson M, Linse S. Protein reconstitution and 3D domain swapping. Curr Protein Pept Sci, 2002, 3: 629-642

8 Schmidt-Rose T, Jentsch T J. Reconstitution of functional voltage-gated chloride channels from complementary fragments of CLC-1. J Biol Chem, 1997, 272: 20515-20521

9 Steinrucken H C, Amrhein N. The herbicide glyphosate is a potent inhibitor of 5-enolpyruvyl-shikimic acid-3-phosphate synthase. Biochem Biophys Res Commun, 1980, 94: 1207-1212

10 Bentley R. The shikimate pathway-A metabolic tree with many branches. Crit Rev Biochem Mol Biol, 1990, 25: 307-384

11 Schonbrunn E, Eschenburg S, Shuttleworth W A, et al. Interaction of the herbicide glyphosate with its target enzyme 5-enolpyruvylshikimate 3-phosphate synthase in atomic detail. Proc Natl Acad Sci USA, 2001, 98: 1376-1380 
12 Padgette S R, Re D B, Gasser C S, et al. Site-directed mutagenesis of a conserved region of the 5-enolpyruvylshikimate-3-phosphate synthase active site. J Biol Chem, 1991, 266: 22364-22369

13 Eschenburg S, Healy M L, Priestman M A, et al. How the mutation glycine 96 to alanine confers glyphosate insensitivity to 5-enolpyruvyl shikimate-3-phosphate synthase from Escherichia coli. Planta, 2002, 216: $129-135$

14 Stallings W C, Abdel-Meguid S S, Lim L W, et al. Structure and topological symmetry of the glyphosate target 5-enolpyruvylshikimate-3-phosphate synthase: A distinctive protein fold. Proc Natl Acad Sci USA, 1991, 88: 5046-5050

15 Krekel F, Oecking C, Amrhein N, et al. Substrate and inhibitor-induced conformational changes in the structurally related enzymes UDP-N-acetylglucosamine enolpyruvyl transferase (MurA) and 5-enolpyruvylshikimate 3-phosphate synthase (EPSPS). Biochemistry, 1999, 38: 8864-8878

16 Sun Y C, Li Y, Zhang H, et al. Reconstitution of the enzyme AroA and its glyphosate tolerance by fragment complementation. FEBS Lett, 2006, 580: 1521-1527

17 Sun Y C, Chen Y C, Tian Z X, et al. Novel AroA with high tolerance to glyphosate, encoded by a gene of Pseudomonas putida 4G-1 isolated from an extremely polluted environment in China. Appl Environ Microbiol, 2005, 71: 4771-4776

18 Link A J, Phillips D, Church G M. Methods for generating precise deletions and insertions in the genome of wild-type Escherichia coli: application to open reading frame characterization. J Bacteriol, 1997, 179: 6228-6237

19 Pittard A J. TyrR protein of Escherichia coli and its role as repressor and activator. In: Neidhardt F C, Curtiss R III, Ingraham J L, et al., eds. Escherichia coli and Salmonella: Cellular and Molecular Biology, 2nd ed. Washington DC: American Society for Microbiology, 1996. 458-484

20 Gottesman S. Proteolysis in bacterial regulatory circuits. Annu Rev Cell Dev Biol, 2003, 19: 565-587

21 Berggard T, Julenius K, Ogard A, et al. Fragment complementation studies of protein stabilization by hydrophobic core residues. Biochemistry, 2001, 40: 1257-1264

22 Xue W F, Szczepankiewicz O, Bauer M C, et al. Intraversus intermolecular interactions in monellin: Contribution of surface charges to protein assembly. J Mol Biol, 2006, 358: 1244-1255

23 Dell'Orco D, Xue W F, Thulin E, et al. Electrostatic contributions to the kinetics and thermodynamics of protein assembly. Biophys $\mathrm{J}$, 2005, 88: 1991-2002

24 Schwehm J M, Fitch C A, Dang B N, et al. Changes in stability upon charge reversal and neutralization substitution in staphylococcal nuclease are dominated by favorable electrostatic effects. Biochemistry, 2003, 42: 1118-1128

25 Ojennus D D, Lehto S E, Wuttke D S. Electrostatic interactions in the reconstitution of an $\mathrm{SH} 2$ domain from constituent peptide fragments. Protein Sci, 2003, 12: 44-55

Open Access This article is distributed under the terms of the Creative Commons Attribution License which permits any use, distribution, and reproduction in any medium, provided the original author(s) and source are credited. 